\title{
Transcriptomic Analysis of Staphylococcus epidermidis Biofilm-Released Cells upon Interaction with Human Blood Circulating Immune Cells and Soluble Factors
}

\section{OPEN ACCESS}

Edited by:

Alexandre Morrot

Federal University of Rio de Janeiro,

Brazi

Reviewed by:

Pietro Speziale,

University of Pavia, Italy

Sharon Kleinschmidt,

Queensland University of Technology,

Australia

Leah Cole,

Sanofi Pasteur, USA

*Correspondence:

Angela França

afranca@deb.uminho.pt

Specialty section: This article was submitted to Microbial Immunology, a section of the journal

Frontiers in Microbiology

Received: 22 May 2016

Accepted: 07 July 2016

Published: 21 July 2016

Citation:

França A, Pier GB, Vilanova M and Cerca N (2016) Transcriptomic

Analysis of Staphylococcus epidermidis Biofilm-Released Cells upon Interaction with Human Blood

Circulating Immune Cells and Soluble

Factors. Front. Microbiol. 7:1143.

doi: 10.3389/fmicb.2016.01143

\begin{abstract}
Angela França ${ }^{1 *}$, Gerald B. Pier ${ }^{2}$, Manuel Vilanova ${ }^{3,4,5}$ and Nuno Cerca ${ }^{1}$
'Laboratory of Research in Biofilms Rosário Oliveira, Centre of Biological Engineering, University of Minho, Braga, Portugal, ${ }^{2}$ Division of Infectious Diseases, Department of Medicine, Brigham and Women's Hospital/Harvard Medical School, Boston, MA, USA, ${ }^{3}$ Instituto de Ciências Biomédicas de Abel Salazar, Universidade do Porto, Porto, Portugal, ${ }^{4}$ Instituto de Investigação e Inovação em Saúde, Universidade do Porto, Porto, Portugal, ${ }^{5}$ Instituto de Biologia Molecular e Celular, Universidade do Porto, Porto, Portugal
\end{abstract}

Keywords: Staphylococcus epidermidis biofilms, biofilm-released cells, human blood, human plasma, human leukocytes, transcriptome

\section{BACKGROUND}

The colonization of indwelling medical devices by biofilm-forming bacteria is one of the major causes of healthcare-associated infections (Percival et al., 2015). Staphylococcus epidermidis, a biofilm-forming commensal bacterium that inhabits human skin and mucosae, is considered one of most important causes of medical devices-related infections, being particularly associated with the use of intravascular catheters (Mack et al., 2013). Although S. epidermidis biofilms are classically associated with the development of chronic infections (Costerton et al., 1999), the release of cells from the biofilm has been associated with onset of acute infections such as embolic events of endocarditis (Pitz et al., 2011), bacteremia, or even septicemia (Cole et al., 2016). Bloodstream infections caused by S. epidermidis are typically indolent and difficult to eradicate significantly increasing patient's morbidity (Kleinschmidt et al., 2015) and mortality among immunocompromised (Khashu et al., 2006) and immunosuppressed patients (Bender and Hughes, 1980). In addition, the costs associated with the diagnosis and treatment of these secondary infections is estimated to be approximately $\$ 20,000$ per occurrence (Kilgore and Brossette, 2008). Henceforth, it is imperative to redefine strategies for the management of the pathologic events associated with biofilm disassembly. Since bloodstream infections are one of the most frequent complications caused by S. epidermidis biofilm disassembly (Cole et al., 2016), a comprehensive analysis of the interplay between S. epidermidis biofilm-released cells (BRC) and hosts' blood components would be invaluable. Herein, as the first step toward the understanding of this interaction, we have characterized, using RNA sequencing (RNAseq) technology, the transcriptome of S. epidermidis BRC upon interaction with whole human blood, polymorphonuclear, or mononuclear leukocytes and plasma.

\section{MATERIALS AND METHODS}

\section{Ethics Statement}

Human blood was collected from healthy adult volunteers, under a human subject's protocol approved by the Institutional Review Board of the University of Minho (SECVS 002/2014). 
Furthermore, this procedure was performed in agreement with Helsinki declaration and Oviedo convention. All donors gave written consent before blood collection.

\section{Bacteria and Growth Conditions}

S. epidermidis strain 9142, isolated from a blood culture (Mack et al., 1992), was used for this study. BRC were obtained using a fed-batch system in the presence of Tryptic Soy Broth (TSB) supplemented with $0.65 \%$ glucose and under agitation conditions, as detailed elsewhere (França et al., 2016). BRC cells were collected from 12 different originating biofilms and pooled together to decrease the variability inherent to biofilm growth (Sousa et al., 2014). After $10 \mathrm{~s}$ sonication at $33 \%$ amplitude (ColeParmer 750-Watt Ultrasonic Homogenizer 230 VAC, IL, USA), the concentration of $\mathrm{BRC}$ was adjusted to $1 \times 10^{9}$ total cells $/ \mathrm{mL}$, by flow cytometry (EC800, Sony Biotechnology Inc., CA, USA), using SYBR Green (Invitrogen, CA, USA) and propidium iodide (Sigma, MO, USA) staining as previously optimized (Cerca et al., 2011).

\section{Blood Collection and Fractioning}

Peripheral blood was collected into BD Vacutainer ${ }^{\circledR}$ tubes coated with lithium heparin $\left(\mathrm{BD}^{\circledR}, \mathrm{NJ}\right.$, USA). Plasma was separated from the cellular fraction by centrifuging whole blood at $1440 \mathrm{~g}$ for $20 \mathrm{~min}$ at $4^{\circ} \mathrm{C}$. Mononuclear (MN) leukocytes were purified from whole blood using Histopaque 1077 gradient (Sigma) as indicated by the manufacturer. Thereafter, the mononuclear cells-depleted pellet resultant from the Histopaque 1077 gradient was incubated with $1.5 \%(\mathrm{v} / \mathrm{v})$ dextran solution during $35 \mathrm{~min}$, at room temperature, in order to separate polymorphonuclear (PMN) cells from erythrocytes. PMN cells (present in the supernatant) were then transferred in to a new tube and harvested by centrifugation at $450 \mathrm{~g}$ for $15 \mathrm{~min}$ at $4^{\circ} \mathrm{C}$. Both PMN and MN cells were incubated with water for $30 \mathrm{~s}$ to lyse the remaining erythrocytes and, after readjusting the isotonic conditions by adding $10 \times$ PBS, leukocytes were collected by centrifugation at $200 \mathrm{~g}$ for $15 \mathrm{~min}$ at $4^{\circ} \mathrm{C}$. Finally, leukocytes were suspended in $0.5 \mathrm{~mL}$ of donor's plasma and samples purity and viability determined by flow cytometry (EC800, Sony) using, respectively, CD15 (PMN) and CD3 (MN) (eBioscience, CA, USA) and propidium iodide staining ( $5 \mu \mathrm{g} / \mathrm{mL}$, Sigma). Only samples with purity $\geq 90 \%$ and death $\leq 15 \%$ were used. The number of PMN and MN cells was determined also by flow cytometry and the concentration adjusted, in donor's plasma, to $1.0 \times 10^{6}$ cells $/ \mathrm{mL}$.

\section{Co-incubation of Bacteria with Whole Human Blood and Its Circulating Immune Factors}

In $2 \mathrm{~mL}$ tubes, $100 \mu \mathrm{L}$ of a suspension of $1 \times 10^{9}$ total BRC/mL were mixed with $900 \mu \mathrm{L}$ of whole human blood, PMN, or MN at $1.0 \times 10^{6}$ cells $/ \mathrm{mL}$, plasma or TSB (containing the same concentration of heparin as blood and its components) and incubated at $80 \mathrm{rpm}$ (in a $10 \mathrm{~mm}$ orbit incubator), for $2 \mathrm{~h}$ at $37^{\circ} \mathrm{C}$. After the co-incubation period, samples were sonicated for $5 \mathrm{~s}$ at $33 \%$ amplitude (Cole-Parmer) in order to lyse, and thus, decrease eukaryotic cells contamination. Finally, bacteria were harvested by 5 min centrifugation at $16,000 \mathrm{~g}$ at $4^{\circ} \mathrm{C}$ and immediately suspended in $1 \mathrm{~mL}$ of RNA protect ${ }^{\mathrm{TM}}$ bacteria reagent (QIAGEN, Hilden, Germany), which was diluted 2:1 in nuclease-free water (Gibco, MD, USA). BRC before the co-incubation assays (T0h) were also collected. This assay was performed four independent times using blood of four different donors (both female and male).

\section{RNA Isolation and Libraries Construction for RNA Sequencing Analysis}

Total RNA was isolated using RNeasy mini kit (QIAGEN). Bacterial cell lysis was achieved by mechanical $(3.0 \mathrm{~mm}$ zirconium beads, Sigma) and chemical lysis (phenol, Fisher Scientific, MA, USA) as optimized before (França et al., 2012). RNA quality was assessed using an Experion $^{\mathrm{TM}}$ automated electrophoresis system (Bio-Rad, CA, USA). RNA quality indicators were above 9 for all samples. Total RNA purified from each of the four independent coincubation assays performed were pooled together to decrease donor-associated variability. Subsequently, pooled RNA was treated with TURBO DNase (Ambion, NY, USA) and acidphenol:chloroform:isoamyl alcohol (125:24:1) (Ambion, MA, USA) to degrade and isolate, respectively, contaminating genomic DNA. Potential contaminating eukaryotic RNA was removed using MICROBEnrich ${ }^{\mathrm{TM}}$ kit (Ambion). Prokaryotic messenger RNA was then enriched by depleting ribosomal RNA using Ribo-Zero ${ }^{\mathrm{TM}}$ rRNA removal kit for Gram-positive bacteria (Illumina, CA, USA) and transcriptomic libraries were constructed using ScriptSeq ${ }^{\mathrm{TM}}$ RNA-seq library preparation kit (Illumina). The quality of the libraries constructed for each condition under study was assessed by quantitative PCR and Hi-Sensitivity D1K TapeStation (Agilent 2200 TapeStation). Finally, all libraries were multiplexed and sequencing data was generated in a MiSeq ${ }^{\circledR}$ sequencer (Illumina) from paired-end reads $(2 \times 150 \mathrm{bp})$.

The results obtained were validated by quantitative (q) PCR, as described earlier (Sousa et al., 2014). Total RNA samples utilized for RNAseq analysis were used as template. The sequences of the primers used are shown in Supplementary Table 1. Fold-change values were determined applying the Pfaffl method (Pfaffl, 2004) using T0h as control.

\section{Trimming and Data Analysis}

After sequencing, adapters were trimmed by MiSeq ${ }^{\circledR}$ internal software during the base calling. CLC Genomics Workbench version 5.1 (QIAGEN) was then used for quality, ambiguity, and length trimming, alignment with S. epidermidis RP62A (GenBank accession number: CP000029.1), normalization of the reads per kilobase per million mapped reads (Mortazavi et al., 2008), and for the analysis of differential gene expression. Quality, ambiguity, and length trimming were performed using the CLC genomics workbench default settings. The transcriptomic alterations induced on BRC incubated with human blood or its circulating immune cells and soluble factors was determined using as control BRC transcriptomic profile before co-incubation assays. Kal's statistical test (Kal et al., 1999), with false discovery rate (FDR) (Pawitan et al., 2005), 
was applied to identify statistically significant alterations in gene transcription. Alterations with fold changes below 3 and $P$ values above 0.05 were discarded. Taking into consideration that bacteria may change their transcriptome, in a nonspecific manner, after being transferred in to a new environment, we had also compared the transcriptomic alterations observed in BRC incubated with whole blood or its cellular and soluble components with those observed in the presence of $\mathrm{TSB}$, a standard laboratorial medium. Hence, in order to only consider alterations induced by biological factors, genes whose transcription was found in both groups were discarded. Nevertheless, genes with opposite regulation in both groups were maintained. KEGG analysis was performed using STRING (Franceschini et al., 2013) and only gene-sets passing significance thresholds $(P<0.05$, Hypergeometric test with FDR) are depicted. The heat map was created using CIMminer utilizing Euclidean distance method and average linkage clustering.

\section{DATA DEPOSITION}

The transcriptomic profile of S. epidermidis BRC under the conditions described in this study was deposited in Gene Expression Omnibus (GEO) database, at NCBI, under the accession number GSE79948. Raw and trimmed/filtered datasets are available and can be downloaded as fastq and text files, respectively.

\section{INTERPRETATION OF DATA SETS}

Bacteremia is one of the major clinical complications associated with the release of cells from S. epidermidis biofilms formed on intravascular catheters. These infections are associated with increased hospitalization periods, healthcare costs and patient morbidity, or even mortality (Kleinschmidt et al., 2015). Our goal was thus to gather the first insights into the interaction

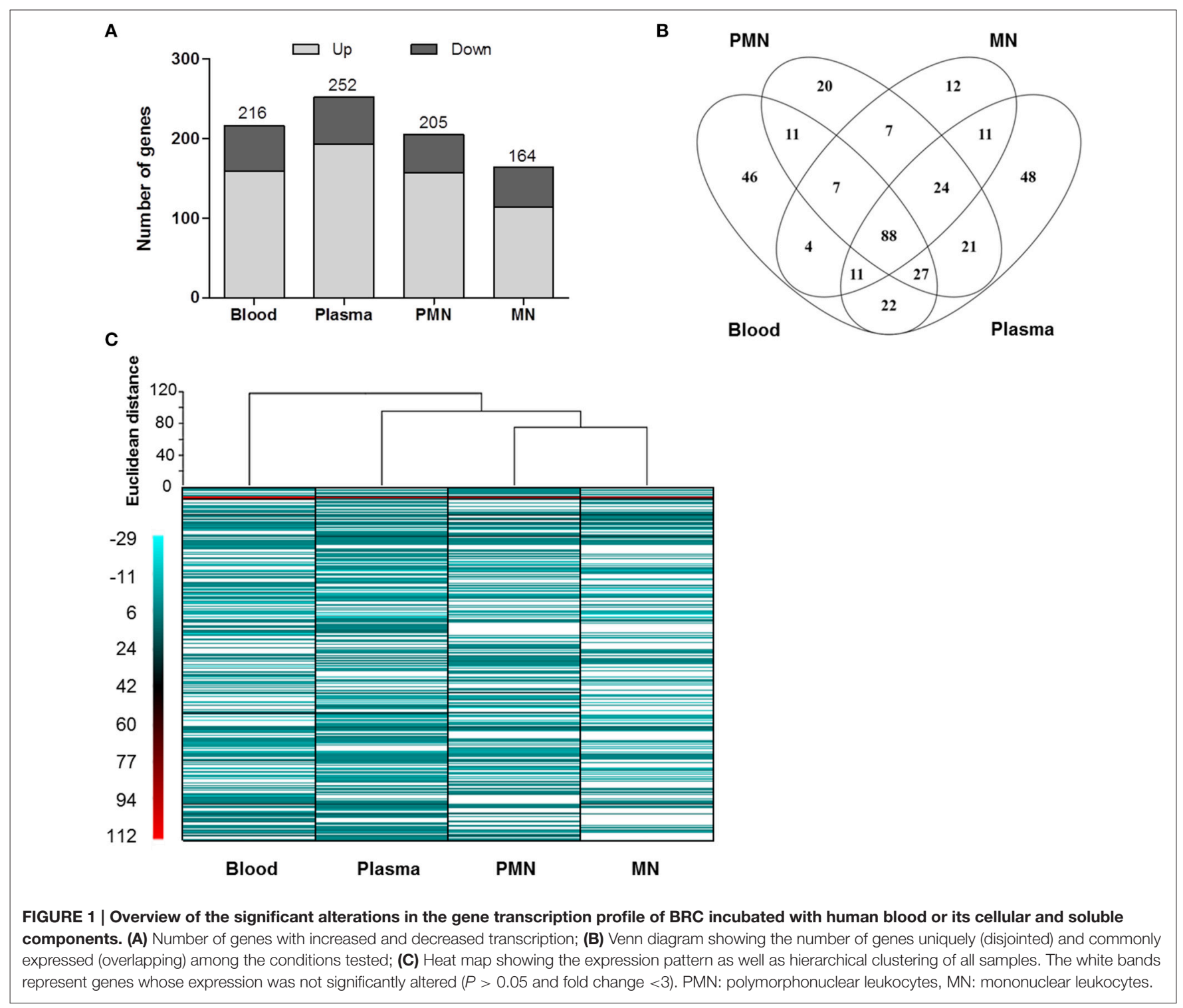


between S. epidermidis BRC and human blood, in order to better understand the role of these cells in the pathogenesis of $S$. epidermidis biofilm-related infections. To this end, we analyzed the transcriptome of BRC upon interaction with human blood or its cellular and soluble components, using an ex vivo model previously used for other microorganisms (Fradin et al., 2003; Mereghetti et al., 2008; Malachowa et al., 2011; França et al., 2014).

The transcriptome of S. epidermidis BRC changed significantly after incubation with whole human blood or its cellular or soluble components. Nevertheless, before analyzing the alterations observed, RNAseq results were confirmed using qPCR. As can be seen in Supplementary Figure 1, a strong correlation between both methods was observed. Thereafter, we compared the transcriptome of BRC incubated with human blood, PMNs,
MNs, or plasma with the one of BRC incubated with TSB, a rich medium frequently used in in vitro assays. This comparison allowed the identification the genes whose transcription was affected only by the presence of biological factors. Within the genes found differentially transcribed (fold change $>3$ and $P<$ 0.05 , Kal's test with FDR) only $21-25 \%$ of the genes were found in BRC incubated with whole human blood or its cellular and soluble components and these were the genes considered for further analysis (Figure 1).

KEGG pathways analysis showed that the major alterations occurring in the presence of human blood circulating factors were associated with basic pathways involved in the biosynthesis or metabolism of amino acids and in the import or export of substances (Figure 2). In addition, KEGG pathways such as biosynthesis of secondary metabolites and phenylalanine,

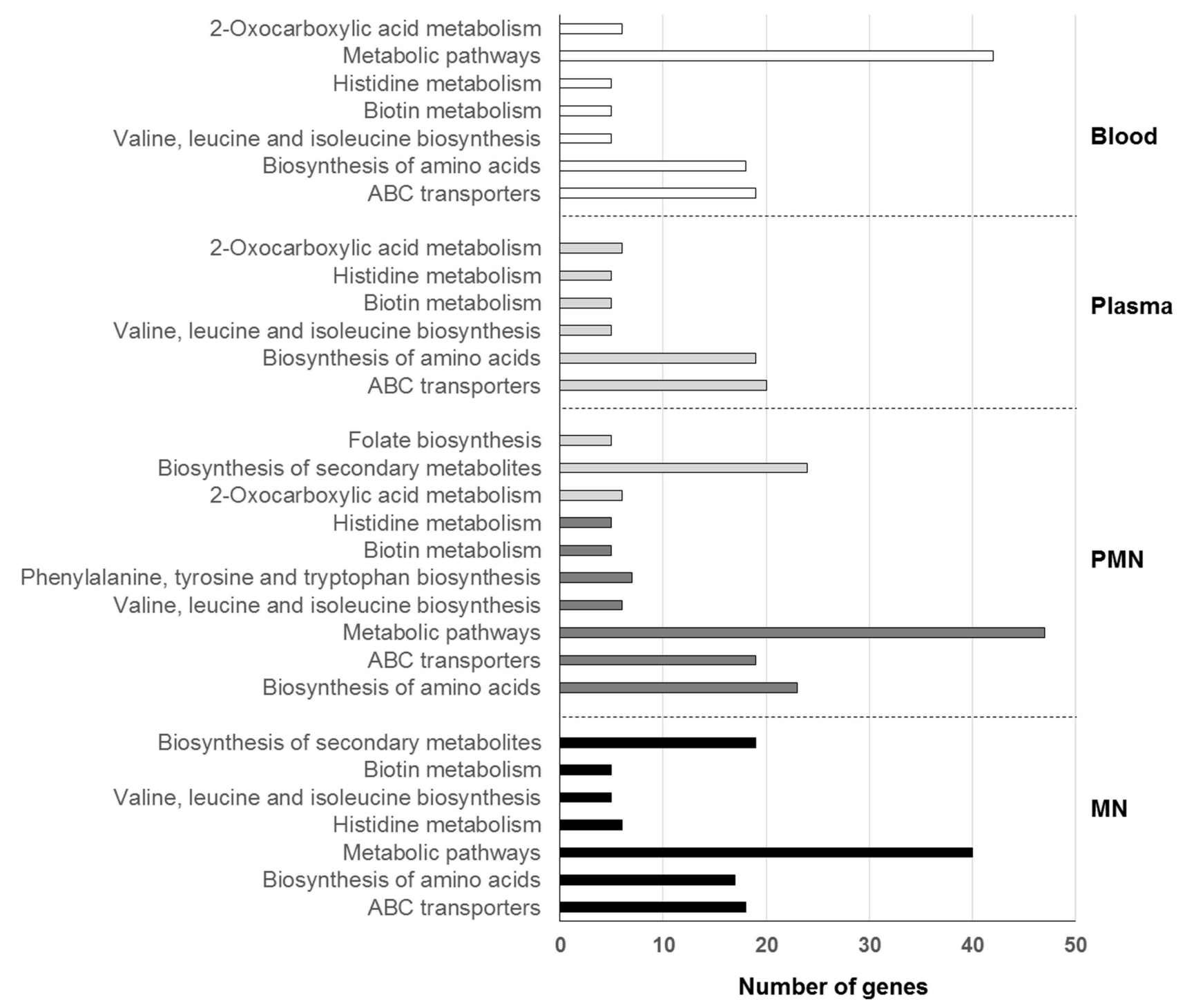

FIGURE 2 | KEGG pathways enriched after incubation with whole human blood or its cellular or soluble components. KEEG pathways are organized, for each condition, from lower (top) to higher (bottom) significance. PMN, polymorphonuclear leukocytes; MN, mononuclear leukocytes. 
tyrosine, tryptophan, and folate biosynthesis were also found significantly enriched $(P<0.05$, Hypergeometric test with FDR) but only in BRC incubated with both PMN and MN leukocytes (Figure 2).

As it would be expected in a low free iron environment such as human blood/plasma, iron transferrin receptors, iron $\mathrm{ABC}$ transporter permeases, and iron $\mathrm{ABC}$ transporter ATP binding proteins were found significantly enriched. Interestingly, biotin metabolism was also found significantly enriched $(P<0.05$, Hypergeometric test with FDR) in all the conditions tested. In fact, the complete biotin operon, which is composed by the genes bioA, bioD, bioF, and bioW, was found highly transcribed, with fold change values ranging from 13 to 110 . The same observation was made in $S$. aureus after short-term incubation with either blood or plasma (Malachowa et al., 2011). Biotin is an important cofactor involved in prokaryotic central pathways being particularly important during infection, as bacteria have a high demand for micronutrients (Streit and Entcheva, 2003). Due to the essential nature of biotin metabolism, the target of biotin metabolism-associated proteins has been under special attention, being considered a promising strategy to combat drugresistant pathogens including S. aureus (Soares da Costa et al., 2012; Pendini et al., 2013; Paparella et al., 2014). No enrichment was found among genes with decreased transcription. Interestingly, in all the conditions tested, $\sim 40 \%$ of the genes with down-regulated transcription encoded hypothetical proteins or pseudogenes.

Analyzing the effect of each of the blood components independently, using hierarchical cluster analysis, we found two major clades: one that separates BRC incubated with whole human blood from the remaining conditions and a second clade that separates BRC incubated with plasma from those incubated with either PMN or $\mathrm{MN}$ cells (Figure 2). Interestingly, this suggested that both PMN and MN leukocytes, despite their involvement in innate immunity and phagocytic capability, had little influence on $S$. epidermidis BRC gene expression profile. Also, these results indicated that the majority of the alterations observed in whole human blood occurred in plasma, as recently observed in a small panel of $S$. epidermidis genes (França and Cerca, 2016). We cannot, however, discard the possibility that a different effect on S. epidermidis gene expression profile could be seen if these cells were circulating in human blood, as crosstalk

\section{REFERENCES}

Bender, J. W., and Hughes, W. T. (1980). Fatal Staphylococcus epidermidis sepsis following bone marrow transplantation. Johns Hopkins Med. J. 146, 13-15.

Cerca, F., Trigo, G., Correia, A., Cerca, N., Azeredo, J., and Vilanova, M. (2011). SYBR green as a fluorescent probe to evaluate the biofilm physiological state of Staphylococcus epidermidis, using flow cytometry. Can. J. Microbiol. 57, 850-856. doi: 10.1139/w11-078

Cole, L. E., Zhang, J., Kesselly, A., Anosova, N. G., Lam, H., Kleanthous, H., et al. (2016). Limitations of murine models for assessment of antibody-mediated therapies or vaccine candidates against $S$. epidermidis bloodstream infection. Infect. Immun. 4, 1143-1149. doi: 10.1128/IAI.01472-15 with and production of signaling molecules by other cells may have an important influence in S. epidermidis gene expression.

\section{CONCLUSIONS}

Overall, this study enabled us to identify the pathways involved in the adaptation of the bacterium to the stressful environment encountered in human blood consequently contributing to its survival and persistence. Hence, these results may be helpful in the selection of potential targets for future studies aiming to develop preventive and/or therapeutic strategies for $S$. epidermidis biofilm-based infections. One interesting target may be biotin metabolism-associated proteins, which are already being tackled in other important human pathogens.

\section{AUTHOR CONTRIBUTIONS}

GP, MV, and NC conceived the study and participated in its design and coordination. AF performed the experiments, collected, analyzed, and deposited the data. AF prepared the draft and GP, MV, and NC proofread the final draft. All authors have read and approved the manuscript.

\section{FUNDING}

This study was funded by the Portuguese Foundation for Science and Technology (FCT) by the project with the reference FCOMP-01-012014-FEDER-041246 (EXPL/BIAMIC/0101/2013), the strategic funding of UID/BIO/04469/2013 unit, COMPETE 2020 (POCI-01-0145-FEDER-006684), BioTecNorte operation (NORTE-01-0145-FEDER-000004) funded by European Regional Development Fund under the scope of Norte2020 - Programa Operacional Regional do Norte. NC is an Investigador FCT. AF is supported by the FCT fellowship SFRH/BPD/99961/2014. The funders had no role in study design, data collection and interpretation, or decision to submit the work for publication.

\section{SUPPLEMENTARY MATERIAL}

The Supplementary Material for this article can be found online at: http://journal.frontiersin.org/article/10.3389/fmicb. 2016.01143 
França, A., and Cerca, N. (2016). Plasma is the main regulator of Staphylococcus epidermidis biofilms virulence genes transcription in human blood. Pathog. Dis. 74:ftv125. doi: 10.1093/femspd/ftv125

França, A., Freitas, A. I., Henriques, A. F., and Cerca, N. (2012). Optimizing a $\mathrm{qPCR}$ gene expression quantification assay for S. epidermidis biofilms: a comparison between commercial kits and a customized protocol. PLoS ONE 7:e37480. doi: 10.1371/journal.pone.0037480

Franceschini, A., Szklarczyk, D., Frankild, S., Kuhn, M., Simonovic, M., Roth, A., et al. (2013). STRING v9.1: protein-protein interaction networks, with increased coverage and integration. Nucleic Acids Res. 41, D808-D815. doi: $10.1093 /$ nar/gks1094

Kal, A. J., van Zonneveld, A. J., Benes, V., van den, B. M., Koerkamp, M. G., Albermann, K., et al. (1999). Dynamics of gene expression revealed by comparison of serial analysis of gene expression transcript profiles from yeast grown on two different carbon sources. Mol. Biol. Cell 10, 1859-1872. doi: 10.1091/mbc.10.6.1859

Khashu, M., Osiovich, H., Henry, D., Al Khotani, A., Solimano, A., and Speert, D. P. (2006). Persistent bacteremia and severe thrombocytopenia caused by coagulase-negative Staphylococcus in a neonatal intensive care unit. Pediatrics 117, 340-348. doi: 10.1542/peds.2005-0333

Kilgore, M., and Brossette, S. (2008). Cost of bloodstream infections. Am. J. Infect. Control 36, S172-S173. doi: 10.1016/j.ajic.2008.10.004

Kleinschmidt, S., Huygens, F., Faoagali, J., Rathnayake, I. U., and Hafner, L. M. (2015). Staphylococcus epidermidis as a cause of bacteremia. Future Microbiol. 10, 1859-1879. doi: 10.2217/fmb.15.98

Mack, D., Davies, A. P., Harris, L. G., Jeeves, R., Pascoe, B., Knobloch, J. K., et al. (2013). "Staphylococcus epidermidis in biomaterial associated infections," in Biomaterials Associated Infection: Immunological Aspects and Antimicrobial Strategies, eds T. F. Moriarty, A. J. S. Zaat, and H. J. Busscher (New York, NY: Springer Science + Business Media), 25-56.

Mack, D., Siemssen, N., and Laufs, R. (1992). Parallel induction by glucose of adherence and a polysaccharide antigen specific for plastic-adherent Staphylococcus epidermidis: evidence for functional relation to intercellular adhesion. Infect. Immun. 60, 2048-2057.

Malachowa, N., Whitney, A. R., Kobayashi, S. D., Sturdevant, D. E., Kennedy, A. D., Braughton, K. R., et al. (2011). Global changes in Staphylococcus aureus gene expression in human blood. PLoS ONE 6:e18617. doi: 10.1371/journal.pone.0018617

Mereghetti, L., Sitkiewicz, I., Green, N. M., and Musser, J. M. (2008). Extensive adaptive changes occur in the transcriptome of Streptococcus agalactiae (group B streptococcus) in response to incubation with human blood. PLoS ONE 3:e3143. doi: 10.1371/journal.pone.0003143

Mortazavi, A., Williams, B. A., McCue, K., Schaeffer, L., and Wold, B. (2008). Mapping and quantifying mammalian transcriptomes by RNA-Seq. Nat. Methods 5, 621-628. doi: 10.1038/nmeth.1226
Paparella, A. S., Soares da Costa, T. P., Yap, M. Y., Tieu, W., Wilce, M. C., Booker, G. W., et al. (2014). Structure guided design of biotin protein ligase inhibitors for antibiotic discovery. Curr. Top. Med. Chem. 14, 4-20. doi: 10.2174/1568026613666131111103149

Pawitan, Y., Michiels, S., Koscielny, S., Gusnanto, A., and Ploner, A. (2005). False discovery rate, sensitivity and sample size for microarray studies. Bioinformatics 21, 3017-3024. doi: 10.1093/bioinformatics/bti448

Pendini, N. R., Yap, M. Y., Traore, D. A., Polyak, S. W., Cowieson, N. P., Abell, A., et al. (2013). Structural characterization of Staphylococcus aureus biotin protein ligase and interaction partners: an antibiotic target. Protein Sci. 22, 762-773. doi: $10.1002 /$ pro. 2262

Percival, S. L., Suleman, L., Vuotto, C., and Donelli, G. (2015). Healthcareassociated infections, medical devices and biofilms: risk, tolerance and control. J. Med. Microbiol. 64, 323-334. doi: 10.1099/jmm.0.0 00032

Pfaffl, M. W. (2004). "Quantification strategies in real-time PCR," in $A-Z$ of Quantitative PCR, ed S. A. Bustin (La Jolla, CA: International University Line), 87-112.

Pitz, A. M., Yu, F., Hermsen, E. D., Rupp, M. E., Fey, P. D., and Olsen, K. M. (2011). Vancomycin susceptibility trends and prevalence of heterogeneous vancomycin-intermediate Staphylococcus aureus in clinical methicillin-resistant S. aureus isolates. J. Clin. Microbiol. 49, 269-274. doi: 10.1128/JCM.00914-10

Soares da Costa, T. P., Tieu, W., Yap, M. Y., Pendini, N. R., Polyak, S. W., Sejer, P. D., et al. (2012). Selective inhibition of biotin protein ligase from Staphylococcus aureus. J. Biol. Chem. 287, 17823-17832. doi: 10.1074/jbc.M112.3 56576

Sousa, C., Franca, A., and Cerca, N. (2014). Assessing and reducing sources of gene expression variability in Staphylococcus epidermidis biofilms. Biotechniques 57, 295-301. doi: 10.2144/000114238

Streit, W. R., and Entcheva, P. (2003). Biotin in microbes, the genes involved in its biosynthesis, its biochemical role and perspectives for biotechnological production. Appl. Microbiol. Biotechnol. 61, 21-31. doi: 10.1007/s00253-0021186-2

Conflict of Interest Statement: The authors declare that the research was conducted in the absence of any commercial or financial relationships that could be construed as a potential conflict of interest.

Copyright (c) 2016 França, Pier, Vilanova and Cerca. This is an open-access article distributed under the terms of the Creative Commons Attribution License (CC BY). The use, distribution or reproduction in other forums is permitted, provided the original author(s) or licensor are credited and that the original publication in this journal is cited, in accordance with accepted academic practice. No use, distribution or reproduction is permitted which does not comply with these terms. 